\title{
A Wideband Circular Polarized Antenna using Coplanar Waveguide
}

\author{
Mohammad Imroz Khan, Telagathoti Pitchaiah
}

\begin{abstract}
A wideband coplanar waveguide (CPW) antenna with circular polarization characteristics using modified ground slot is studied in this work. Proposed design incorporates a hexagonal slot instead of rectangular slot, accounting for enhanced impedance matching. This ground slot is energized by a $50 \Omega$ feed line, resulting in excitation of two orthogonal phase quadrature modes (even-odd modes). Thus, fulfilling the essential criteria required for realizing circular polarization. A narrow horizontal slit (lg) is embedded in the ground plane at immediate left of feed line, accounting for wideband characteristics. 10dB impedance bandwidth of proposed antenna extends from 2.65-5.60 $\mathrm{GHz}$, while 3dB axial ratio bandwidth extends from 3.90- $5.80 \mathrm{GHz}$. Hence, overlapping bandwidth of proposed antenna extends from 3.90 - $5.60 \mathrm{GHz}$. RHCP characteristics with monopole radiation pattern makes proposed antenna useful for WLAN, radio navigation and radiolocation applications.
\end{abstract}

Keywords - cpw, circular polarized, slot, wideband, MMIC, RHCP.

\section{INTRODUCTION}

Wideband circular polarized antenna supports high data rate communication, with immunity over multipath fading, Faraday rotation, polarization mismatch loss and adverse weather conditions. Various wideband circular polarized microstrip antennas have been reported in recent past. But their incompatibility with Monolithic Microwave Integrated Circuit (MMIC), limits their applications. So, coplanar waveguides emerged as an alternative planar transmission line which can be used in integrated circuits [1] in place of microstrip lines. Various wideband circular polarized coplanar waveguide antennas with rectangular slot and square slot have been reported. Open ended cross slot is used in [2], parasitic patch with capacitive coupling is used in [3], circular slot with grounded stubs is used in [4], square slot with unbalanced feed line is implemented in [5], open slot with rectangular patch is studied in [6], for realizing circular polarization performance using coplanar waveguides. Concept of generating circular polarization using inductive coupling is first time introduced in [7]. This technique of inductive coupling for realizing circular polarization has been used in this document.

In this document, wideband circular polarization is achieved using coplanar waveguide. Presented antenna design incorporates a hexagonal shaped slot instead of rectangular slot or square slot. Tapered ground slot avoids sudden change of antenna impedance, thereby resulting in

Revised Manuscript Received on April 12, 2019.

Mohammad Imroz Khan, Department of Electronics and Communication Engineering, Vignan's Foundation for Science, Technology and Research, Guntur, Andhra Pradesh, India- 522213. (E-mail: iitdhanbad.imroz@gmail.com

TelagathotiPitchaiah, Department of Electronics and Communication Engineering, Vignan's Foundation for Science, Technology and Research, Guntur, Andhra Pradesh, India- 522213. (E-mail: tp1977_ece@yahoo.com) wideband characteristics. Proposed structure is a righthanded circular polarized antenna with circular polarization bandwidth of $1700 \mathrm{MHz}$.

\section{ANTENNA DESIGN AND ANALYSIS}

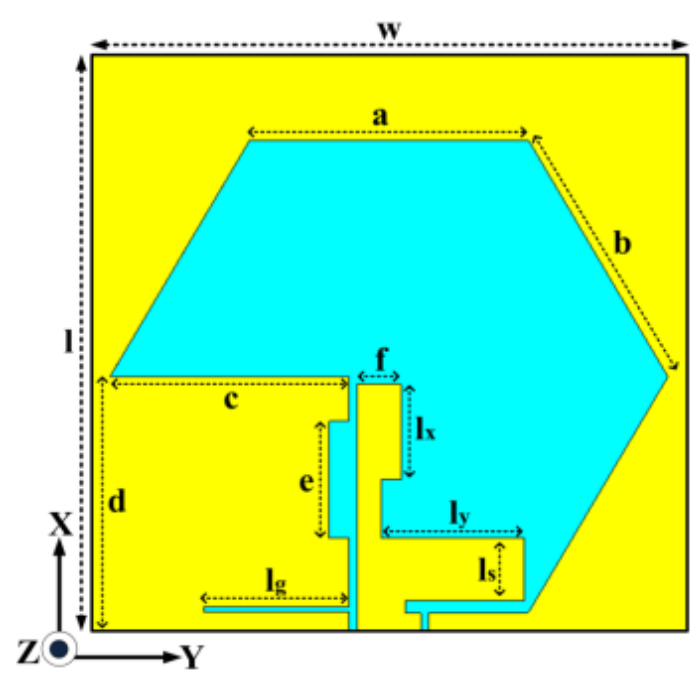

Fig. 1. Top view of proposed antenna

TABLE I. GEOMETRICAL DIMENSIONS

\begin{tabular}{|c|c|c|c|c|c|}
\hline $\mathrm{a}$ & $\mathrm{b}$ & $\mathrm{c}$ & $\mathrm{d}$ & $\mathrm{e}$ & $\mathrm{F}$ \\
\hline 14 & 14 & 12 & 15 & 6 & 3.2 \\
\hline 1 & $1_{\mathrm{g}}$ & $1_{\mathrm{s}}$ & $1_{\mathrm{x}}$ & $1_{\mathrm{y}}$ & $\mathrm{W}$ \\
\hline 30 & 7.3 & 3.2 & 4.9 & 7.2 & 30 \\
\hline
\end{tabular}

Geometrical view of antenna is depicted in fig. 1, while the dimensions of proposed structure is mentioned in table I. Proposed antenna consists of a single side conducting layer, fabricated on FR4 epoxy of thickness $1.6 \mathrm{~mm}$, dielectric strength $=4.4$, loss tangent $=0.02$. A hexagonal shaped slot is embedded in the ground plane and a $50 \Omega$ feed line is inserted into the slot. Circular polarization performance is realized by generating two orthogonal phase quadrature modes.

\section{A. Circular Polarization Analysis}

Orthogonal phase quadrature modes of same magnitude, namely, even mode - odd mode, are excited by inductive coupling in proposed antenna. These even - odd modes are coupled to the ground slot in following set of order: odd $\rightarrow$ even $\rightarrow$ odd $\rightarrow$ even. Magnetic current vectors distribution on feed line and ground plane is depicted in fig. 2. Locus traced by these current vectors confirms circular polarization behavior of antenna (RHCP). The field vectors follow 


\section{A Wideband Circular Polarized Antenna Using Coplanar Waveguide}

anticlockwise sense of rotation in azimuthal plane. Thereby, conforming right-handed circular polarized nature of antenna. Antenna regions responsible for dominant radiations are demonstrated by plotting surface current density at center frequency of operating band, shown in fig. 3 .

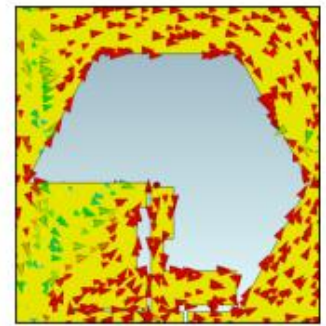

$\omega \mathrm{t}=0^{\circ}$

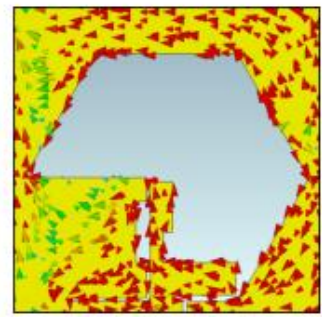

$\omega \mathrm{t}=180^{\circ}$

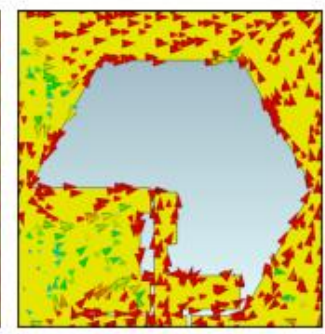

$\omega \mathrm{t}=90^{\circ}$

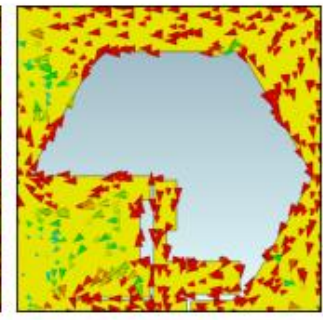

$\omega \mathrm{t}=270^{\circ}$
Fig. 2. Current vector distribution at $4.5 \mathrm{GHz}$

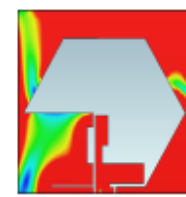

$\omega \mathrm{t}=0^{\circ}$

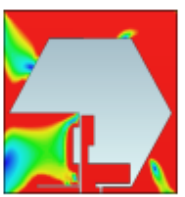

$\omega \mathrm{t}=90^{\circ}$

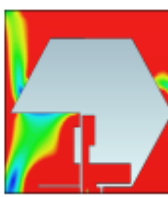

$\omega \mathrm{t}=180^{\circ}$

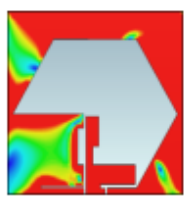

$\omega \mathrm{t}=270^{\circ}$
Fig. 3. Surface current density distribution at $4.5 \mathrm{GHz}$

\section{B. Input Impedance and Antenna Matching}

Matching of input impedance antenna is studied in this section with the help of Smith Chart and VSWR plot, depicted in fig. 4 and fig. 5, respectively. Inductive coupling of antenna is responsible for circular polarization behavior of antenna, confirmed in fig. 4. Input impedance matching is achieved for frequency range extending from 2.65-5.60 $\mathrm{GHz}$, as $\mathrm{VSWR} \leq 2$.

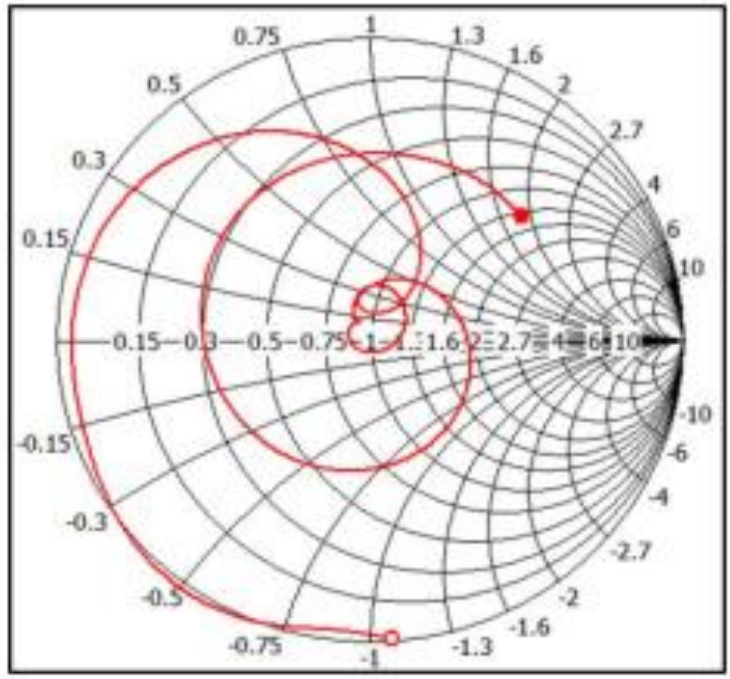

Fig. 4. Smith Chart of antenna

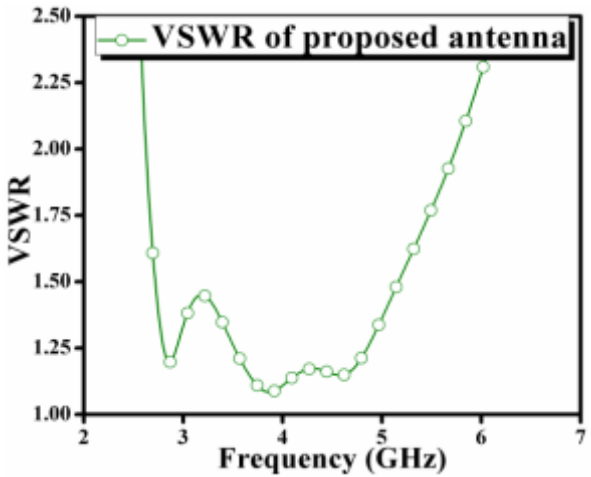

Fig. 5. VSWR of antenna

\section{RESULTS}

S11 (dB) of proposed structure is depicted in fig. 6, which confirms wideband nature of antenna. $10 \mathrm{~dB}$ impedance bandwidth of antenna extends from 2.65-5.60 GHz. CP bandwidth of antenna is illustrated in fig. 7 by the axial ratio plot, which shows that $3 \mathrm{~dB}$ axial ratio bandwidth of antenna extends from 3.90-5.80 GHz. Hence, circular polarization performance of antenna is achieved from $3.90-5.60 \mathrm{GHz}$.

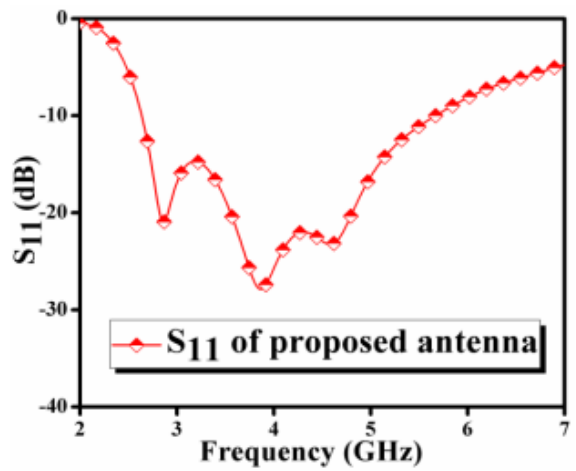

Fig. 6. S11 of proposed antenna

Peak gain of the antenna is $4 \mathrm{dBi}$, which is confirmed in fig. 8. Gain of antenna remains positive for the entire operating band. Efficiency of antenna within the operating band remains more than $85 \%$, confirmed in fig. 9. RHCP and LHCP radiation patterns of antenna at 4.5 and $5.5 \mathrm{GHz}$ are plotted in fig. 10 and fig. 11, respectively. From radiation pattern plots it is evident that the antenna shows RHCP nature with monopole radiation pattern.

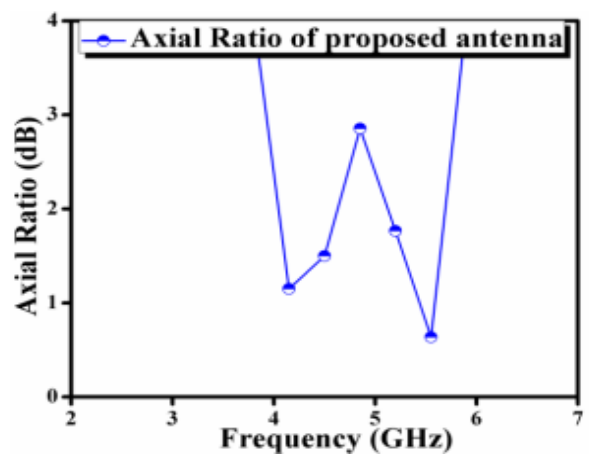

Fig. 7. Axial ratio of proposed antenna 


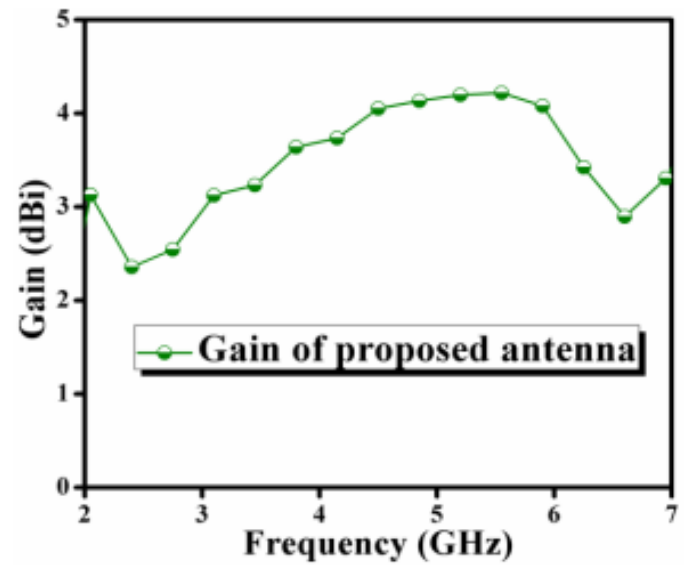

Fig. 8. Gain of proposed antenna

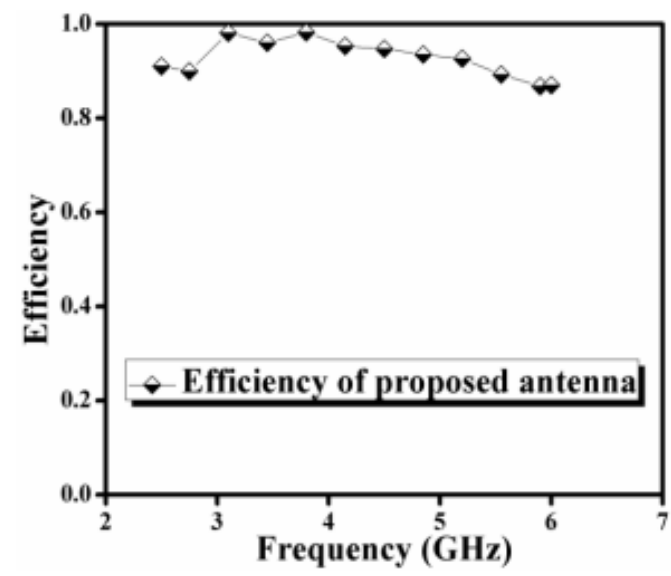

Fig. 9. Efficiency of antenna

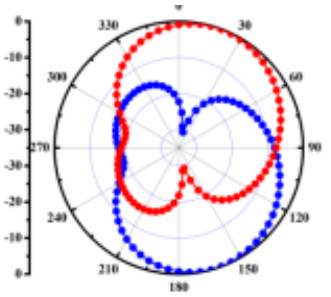

(i)

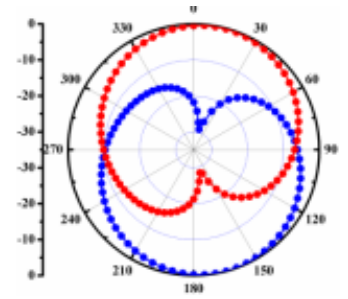

(ii)
Fig. 10. Radiation pattern at 4.5 GHz: (i) XZ plane, (ii) YZ plane

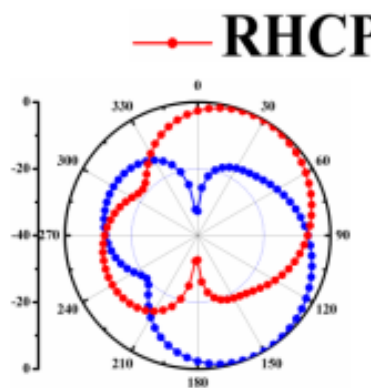

(i)

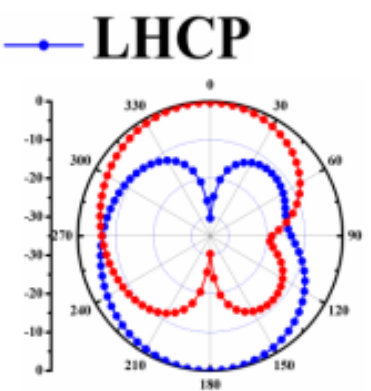

(ii)
Fig. 11. Radiation pattern at $5.5 \mathrm{GHz}$ (i) $\mathrm{XZ}$ plane, (ii) YZ plane
TABLE II. COMPARISON TABLE

\begin{tabular}{|c|c|c|}
\hline References & Dimensions $(1 \times \mathrm{w})$ & $\begin{array}{c}3 \mathrm{~dB} \text { Axial Ratio } \\
\text { Bandwidth } \\
(\mathrm{MHz})\end{array}$ \\
\hline$[2]$ & $50 \times 60$ & 210 \\
\hline$[3]$ & $100 \times 100$ & 400 \\
\hline$[4]$ & $120 \times 120$ & 332 \\
\hline$[8]$ & $60 \times 50$ & 1400 \\
\hline Proposed & $30 \times 30$ & 1700 \\
\hline
\end{tabular}

\section{CONCLUSION}

A wideband circular polarized coplanar waveguide antenna with quasi hexagonal shaped ground slot has been presented. Tapered ground slot along with horizontal slit $1 \mathrm{~g}$ is responsible for wideband characteristics. Concept of inductive coupling is implemented in this work for realizing circular polarization performance. Circular polarization performance of antenna extends from 3.90-5.60 GHz. Monopole radiation pattern with right-handed circular polarization characteristics makes proposed antenna a strong candidate for WLAN, radio navigation and radiolocation applications, with MMIC compatibility.

\section{REFERENCES}

1. T. C. Edwards and M. B. Steer, "Foundation of interconnect and Microstrip Design," 3rd ed., New York: Wiley,2000.

2. C. J. Wang, C. M. Lin, "A CPW-fed open-slot antenna for multiple wireless communication systems", IEEE Antennas and Wireless Propagation Lett., vol. 11, pp. 620-623, 2012.

3. S.P. Pan, J.-Y. Sze, P.-J. Tu, "Circularly Polarized Square Slot Antenna with a Largely Enhanced Axial-Ratio Bandwidth", IEEE Antennas and Wireless Propagation Letters, vol. 11, pp. 969-972, 2012

4. R. Cao, S. Yu, "Wideband compact CPW-fed circularly polarized antenna for universal UHF RFID reader", IEEE Trans. Antennas Propag., vol. 63, no. 9, pp. 4148-4151, Sep. 2015.

5. Y. W. Liu, P. Hsu, "Broadband circularly polarized square slot antenna fed by coplanar waveguide", Electron. Lett., vol. 49, no. 16, pp. 976- 977, 2013.

6. J. Y. Jan, C. Y. Pan, K. Y. Chiu, H. M. Chen, "Broadband CPW-fed circulary-polarized slot antenna with an open slot", IEEE Trans. Antennas Propag., vol. 61, pp. 1418-1422, 2013.

7. Deng, I. , Lin, R. , Chang, K. and Chen, J. (2006), Study of a circularly polarized CPW-fed inductive square slot antenna. Microw. Opt. Technol. Lett., 48: 1665-1667. doi:10.1002/mop.21711.

8. Saini, R. K. and Dwari, S. (2015), CPW-fed broadband circularly polarized rectangular slot antenna with L-shaped feed line and parasitic elements. Microw. Opt. Technol. Lett., 57: 1788-1794. doi:10.1002/mop.29188. 